\section{Debilitating Claudication in an Ultramarathon Runner: A Case of Iliac Artery Endofibrosis}

Nikhil Parimi, M.D. ${ }^{1}$, Prince Sethi, M.D. ${ }^{2}$, Kirk Hance, M.D. ${ }^{3}$,

Bryan Vopat, M.D. ${ }^{4}$, Jean-Philippe Darche, M.D. ${ }^{5}$,

Kamal Gupta, M.D. ${ }^{2}$

${ }^{1}$ University of Kansas Medical Center,

Department of Internal Medicine, Kansas City, KS

University of Kansas Health System, Kansas City, KS

${ }^{2}$ Department of Cardiovascular Medicine

${ }^{3}$ Division of Vascular Surgery

${ }^{4}$ Department of Orthopedic Surgery and Sports Medicine

${ }^{5}$ Department of Family Medicine

Received April 12, 2020; Accepted for publication.June 8, 2020; Published online Aug. 17, 2020

\section{INTRODUCTION}

External iliac artery endofibrosis is characterized by intimal fibrosis and often presents as a gradual decrease of exercise capacity due to exertional claudication-like symptoms, traditionally of the lower extremities. ' The data on the prevalence of iliac artery endofibrosis is limited to case reports; most describe endurance cyclists as the presenting patient. ${ }^{2-4}$ However, there also have been a few cases reported with high performance athletes, including triathletes and long-distance runners. ${ }^{5,6}$ The etiology of this condition is thought to be secondary to mechanical kinking of the external iliac artery during repetitive deep flexion movements, which is seen in certain endurance sports such as cycling or long distance-running. ${ }^{7}$ This is a case of a 43-year-old previous cyclist, now ultramarathon runner, who presented to clinic with severe and progressive exertional lower extremity pain.

\section{CASE REPORT}

A 43-year-old female presented for severe right-sided exertional lower extremity pain of over one year that resolved with rest. She used to be an avid cyclist but had switched to long distance running a few years earlier. She was running 50 - 75 miles weekly prior to the onset of symptoms. She was not a diabetic or a smoker and did not have any risks for premature atherosclerosis. She underwent extensive investigations to determine the etiology of her symptoms. Electromyography and lumbar spine magnetic resonance imaging were normal. She was referred to the sports medicine service and had a work-up for arterial occlusive disease. A physical exam from a vascular medicine specialist demonstrated normal femoral artery pulses bilaterally with slightly diminished dorsalis pedis and posterior tibial pulses bilaterally. Her physical exam was otherwise unremarkable.

She underwent resting and exercise ankle brachial index (ABI) along with magnetic resonance angiography (MRA) of her lower extremities. Resting ABI was normal. Exercise ABI was reduced critically on her right side at 0.19 and remained low six minutes post exercise at 0.22. Her left side was normal. Duplex ultrasound of lower extremity and MRA did not show any significant vascular abnormality, including popliteal entrapment syndrome. Figures 1 and 2 show images from angiography.

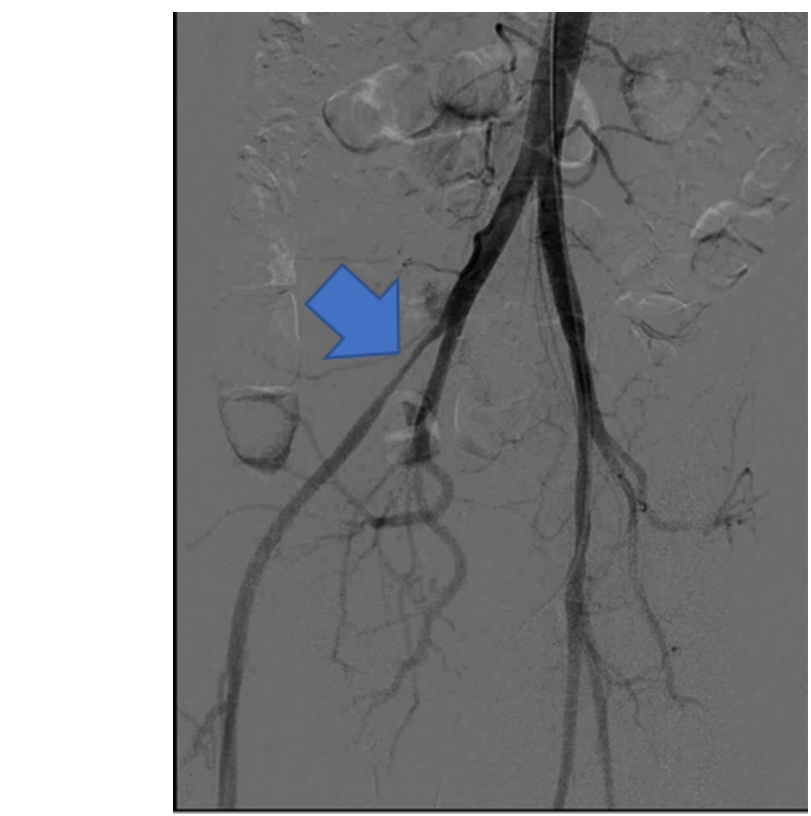

Figure 1. Aortoiliac angiography with peripheral runoff showed $70 \%$ right external iliac stenosis (demonstrated by the arrow) with a gradient of $40 \mathrm{mmHg}$ across the stenosis.

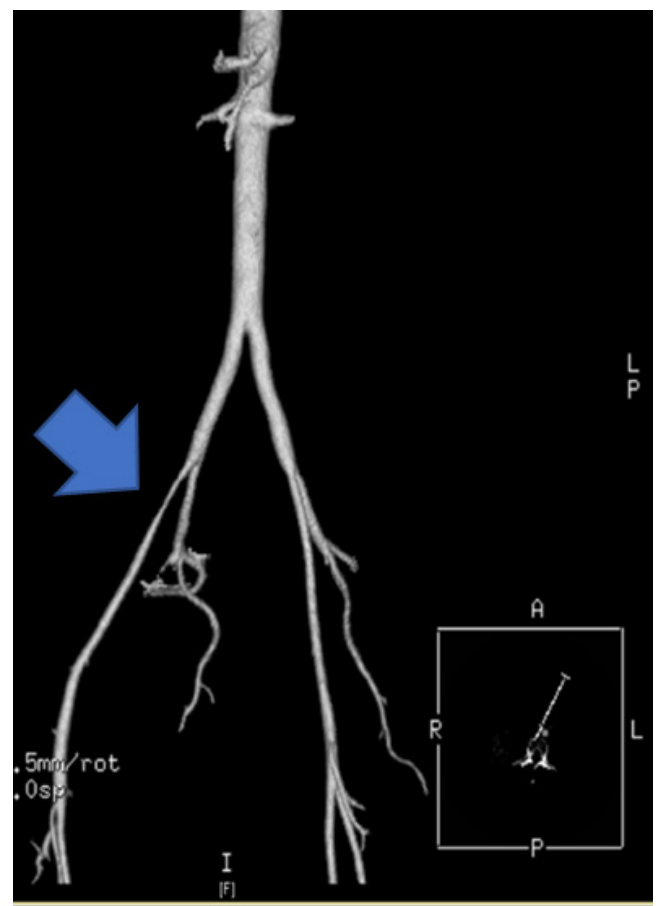

Figure 2. Computed tomography (CT) angiography of the abdomen/pelvis revealed a smooth $70 \%$ stenosis of the right external iliac artery (demonstrated by the arrow) but no atherosclerotic plaque, consistent with endofibrosis.

Her erythrocyte sedimentation rate and c-reactive protein were normal and vasculitis as a cause of her presentation was ruled out. She underwent a successful right external iliac artery endarterectomy with saphenous vein patch (Figures 3 and 4). A specimen of the endarterectomy is shown in Figure 5. She tolerated the procedure well and had improvement in her symptoms. 


\section{KANSAS JOURNAL of MEDICINE}

ILIAC ARTERY ENDOFIBROSIS

continued.

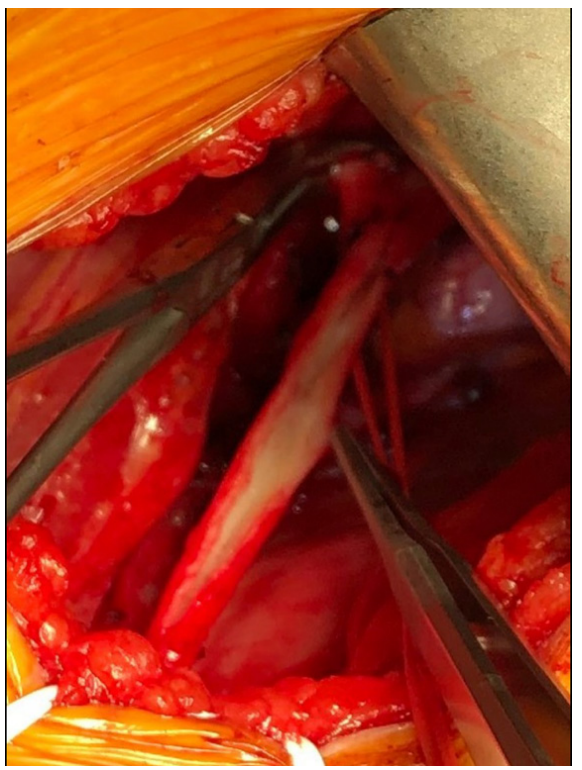

Figure 3. Open right external iliac artery showing the smooth endofibrosis in situ.

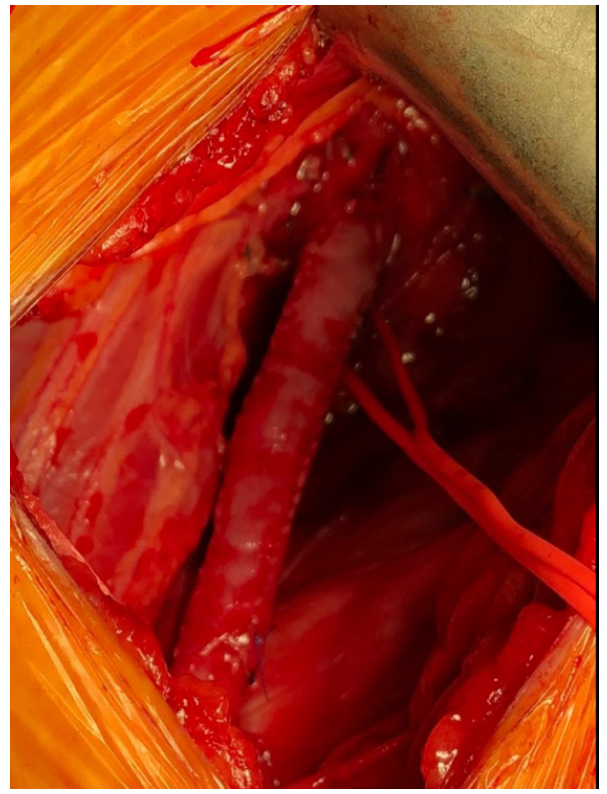

Figure 4. External iliac artery with saphenous vein patch surgical closure.

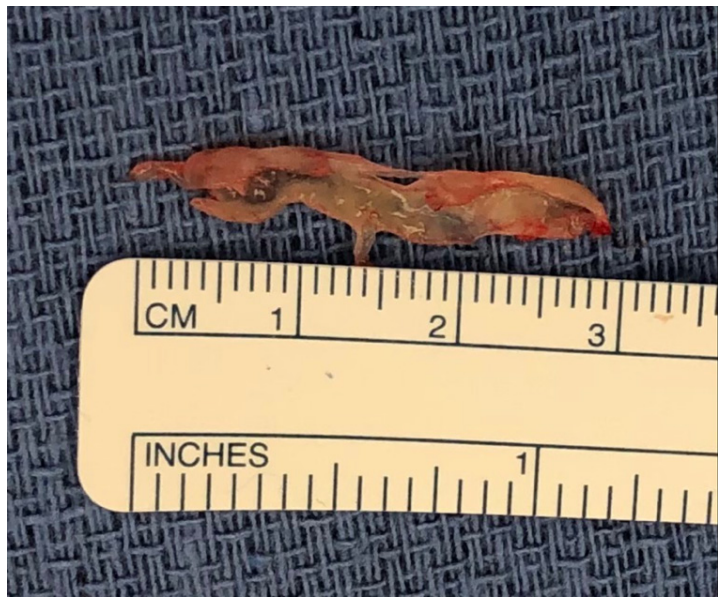

Figure 5. Endarterectomy specimen demonstrating the endofibrosis.

\section{DISCUSSION}

In patients who present with exertional claudication-like symptoms, it is important to distinguish between atherosclerotic and non-atherosclerotic peripheral artery disease. ${ }^{8}$ The clues that suggest atherosclerotic peripheral artery disease are older age, presence of associated cardiovascular risk factors (diabetes, tobacco use, hypertension, hypercholesterolemia), and clinical manifestations or history of atherosclerosis in other vascular beds (myocardial infarction, ischemic stroke). Physical exam changes that can increase the pretest probability of atherosclerotic peripheral artery disease include symmetrically diminished pulses, audible bruit over affected artery, and in severe cases trophic changes to skin and nails.

The pretest probability for atherosclerotic disease in our patient was low. She had undergone evaluation for non-atherosclerotic processes and was diagnosed with external iliac artery endofibrosis. Other causes of non-atherosclerotic arterial occlusive disorders that were considered and excluded in this patient were popliteal artery entrapment syndrome, cystic adventitial disease, thromboangitis oblieterans, vasculitides, and chronic exertional compartment syndrome.

Iliac artery endofibrosis has been described as an entity involving the intima of the artery which is under physical stress but there have been reports of associated medial and adventitial hypertrophy as well. When the presentation is not typical, the diagnosis can be often challenging. As a result, patients who present with these symptoms often are misdiagnosed and can undergo unnecessary testing or procedures leading to frustration for both patients and providers.

Exercise ABI is crucial in the initial workup of these patients as it can identify arterial occlusive disease (including iliac artery endofibrosis) with a sensitivity of $90 \%$ and specificity of $87 \% .^{9}$ Further diagnostic modalities which are useful for defining arterial anatomy are imaging modalities (such as duplex ultrasound, CT arteriography, MRA, and invasive arteriography). The imaging modalities are of importance in planning a management strategy. Patients have tried conservative options, such as reduction in precipitating activity, but this is often not acceptable to patients who engage in athletic activities and are, in fact, predisposed to atherosclerosis. Endovascular procedures such as angioplasty and endoluminal stenting generally are beneficial short term without long term success. Open surgical procedures, such as arterial release, vessel shortening with endofibrosectomy, endarterectomy with patch angioplasty (as in our patient), and interposition grafting, have been used with success where most patients have been able to return to their baseline athletic activity.

\section{CONCLUSION}

Iliac artery endofibrosis should be considered in the differential diagnosis in any active patient who presents with reproducible exertional claudication-like symptoms without the risk factors for atherosclerotic peripheral artery disease. Delay in diagnosis and treatment can cause significant physical and social ramifications for patients. In addition, due to the rarity of the disease, management should include a multi-specialty team of physicians with expertise in sports medicine, vascular medicine, and vascular surgery. 


\section{REFERENCES}

${ }^{1}$ Abraham P, Leftheriotis G, Bourre Y, Chevalier JM, Saumet JL. Echography of external iliac artery endofibrosis in cyclists. Am J Sports Med 1993; 21(6):861-863. PMID: 8291640.

${ }^{2}$ Chevalier JM, Enon B, Walder J, et al. Endofibrosis of the external iliac artery in bicycle racers: An unrecognized pathological state. Ann Vasc Surg 1986; 1(3):297-303. PMID: 3504340.

${ }^{3}$ Shalhub S, Zierler RE, Smith W, Olmsted K, Clowes AW. Vasospasm as a cause for claudication in athletes with external iliac artery endofibrosis. J Vasc Surg 2013; 58(1):105-111. PMID: 23541546.

${ }^{4}$ Bruneau A, Le Faucheur A, Mahe G, Vielle B, Leftheriotis G, Abraham P. Endofibrosis in athletes: Is a simple bedside exercise helpful or sufficient for the diagnosis? Clin J Sport Med 2009; 19(4):282-286. PMID: 19638821.

${ }^{5}$ van Rensburg DCJ, van Rensburg AJ, van Duuren EM, Grant CC. Iliac artery endofibrosis in a middle-aged female long-distance runner. Am J Phys Med Rehabil 2014; 93(12):1100-1103. PMID: 25122104.

${ }^{6}$ Maree AO, Islam MA, Snuderl M, et al. External iliac artery endofibrosis in an amateur runner: Hemodynamic, angiographic, histopathological evaluation and percutaneous revascularization. Vasc Med 2007; 12(3):203-206. PMID: 17848477

7 Peach G, Schep G, Palfreeman R, Beard JD, Thompson MM, Hinchliffe RJ. Endofibrosis and kinking of the iliac arteries in athletes: A systematic review. Eur J Vasc Endovasc Surg 2012; 43(2):208-217. PMID: 22186674.

8 Weinberg I, Jaff MR. Nonatherosclerotic arterial disorders of the lower extremities. Circulation 2012; 126(2):213-222. PMID: 22777664,

9 Abraham P, Bickert S, Vielle B, Chevalier JM, Saumet JL. Pressure measurements at rest and after heavy exercise to detect moderate arterial lesions in athletes. J Vasc Surg. 2001; 33(4):721-727. PMID: 11296323.

Keywords: iliac artery, peripheral artery disease, intermittent claudication, athlete
KANSAS JOURNAL of MEDIC INE ILIAC ARTERY ENDOFIBROSIS

\section{continued.}

